\title{
Glycaemic regulation, appetite and ex vivo oxidative stress in young adults following consumption of high-carbohydrate cereal bars fortified with polyphenol-rich berries
}

\author{
Tracey J. Smith ${ }^{1 *}$, James Philip Karl ${ }^{1}$, Marques A. Wilson ${ }^{1}$, Claire C. Whitney ${ }^{1}$, Ann Barrett ${ }^{2}$, \\ Nicole Favreau Farhadi ${ }^{2}$, Chung-Yen Oliver Chen ${ }^{3,4}$ and Scott J. Montain ${ }^{1}$ \\ ${ }^{1}$ Military Nutrition Division, United States Army Research Institute of Environmental Medicine, Natick, MA O1760, USA \\ ${ }^{2}$ Combat Feeding Directorate, Natick Soldier Research, Development and Engineering Center, Natick, MA O1760, USA \\ 3 Jean Mayer USDA Human Nutrition Research Center on Aging, Tufts University, Boston, MA O2111, USA \\ ${ }^{4}$ Biofortis, Mérieux NutriSciences, Addison, IL 60101, USA
}

(Submitted 4 September 2018 - Final revision received 5 February 2019 - Accepted 13 February 2019)

\section{Abstract}

Consumption of certain berries appears to slow postprandial glucose absorption, attributable to polyphenols, which may benefit exercise and cognition, reduce appetite and/or oxidative stress. This randomised, crossover, placebo-controlled study determined whether polyphenol-rich fruits added to carbohydrate-based foods produce a dose-dependent moderation of postprandial glycaemic, glucoregulatory hormone, appetite and ex vivo oxidative stress responses. Twenty participants (eighteen males/two females; 24 (sD 5) years; BMI: 27 (sD 3) kg/m²) consumed one of five cereal bars (approximately $88 \%$ carbohydrate) containing no fruit ingredients (reference), freeze-dried black raspberries (10 or $20 \%$ total weight; LOW-Rasp and HIGH-Rasp, respectively) and cranberry extract ( 0.5 or $1 \%$ total weight; LOW-Cran and HIGH-Cran), on trials separated by $\geq 5 \mathrm{~d}$. Postprandial peak/nadir from baseline $\left(\Delta_{\max }\right)$ and incremental postprandial AUC over 60 and 180 min for glucose and other biochemistries were measured to examine the dose-dependent effects. Glucose $\mathrm{AUC}_{0-180 \text { min }}$ trended towards being higher ( $43 \%$ ) after $\mathrm{HIGH}$ Rasp $v$. LOW-Rasp $(P=0.06)$, with no glucose differences between the raspberry and reference bars. Relative to reference, HIGH-Rasp resulted in a $17 \%$ lower $\Delta_{\max }$ insulin, $3 \%$ lower C-peptide ( AUC $_{0-60 \mathrm{~min}}$ and $3 \%$ lower glucose-dependent insulinotropic polypeptide (AUC $0-180$ min) $P<0 \cdot 05$. No treatment effects were observed for the cranberry bars regarding glucose and glucoregulatory hormones, nor were there any treatment effects for either berry type regarding ex vivo oxidation, appetite-mediating hormones or appetite. Fortification with freeze-dried black raspberries (approximately $25 \mathrm{~g}$, containing $1.2 \mathrm{~g}$ of polyphenols) seems to slightly improve the glucoregulatory hormone and glycaemic responses to a highcarbohydrate food item in young adults but did not affect appetite or oxidative stress responses at doses or with methods studied herein.

Key words: Polyphenols: Tannins: Glycaemia: Appetite: Oxidative stress: Young adults

A sustained postprandial glycaemic response is advantageous for facilitating physical and cognitive performance ${ }^{(1-5)}$. Working memory and selective attention, for example, were improved in the later postprandial period following consumption of a food item (bread enriched with guar gum) that elicited higher net glucose availability in the postprandial period compared with a reference food item (bread without guar gum). In addition, evidence suggests that consuming moderate glycaemic index foods 1-2 $\mathrm{h}$ before exercise better maintains euglycaemia and maximises carbohydrate oxidation during endurance exercise $v$. high glycaemic index foods which result in lower net glucose availability during the postprandial period ${ }^{(1-3)}$. In addition, slowing glucose absorption prevents postprandial hyperinsulinaemia, which in turn causes a rapid drop in blood glucose to below fasting concentrations. The resulting relative hypoglycaemia initiates a counter-regulatory hormone response that promotes gluconeogenesis, glycogenolysis, free fatty acid release and oxidative stress $^{(6)}$. Given the popularity of foods high in rapidly digested carbohydrate and the advantages of promoting a sustained glycaemic response to promote physical and cognitive performance, there is substantial interest in developing food products that moderate postprandial glycaemic responses ${ }^{(7)}$.

Polyphenols are a heterogeneous group of phytochemicals found in plant-based foods, many of which display anti-oxidant and anti-inflammatory properties, but which are also thought to modulate carbohydrate metabolism ${ }^{(7)}$. In support, some studies

Abbreviations: GIP, glucose-dependent insulinotropic polypeptide; GLP-1, glucagon-like peptide-1; HIGH-Cran, $1 \cdot 0 \%$ cranberry extract cereal bar; HIGH-Rasp, $20 \%$ black raspberry cereal bar; LOW-Cran, $0.5 \%$ cranberry extract cereal bar; LOW-Rasp, $10 \%$ black raspberry cereal bar; USARIEM, U.S. Army Research Institute of Environmental Medicine.

*Corresponding author: T. J. Smith, fax +1 508233 5833, email Tracey.Smith10.civ@mail.mil 
have shown that whole berries, berry extracts, apple extract or juice and a mixture of polyphenol and fibre-rich foods (e.g. green tea, apple peel and freeze-dried berry powders) modulate the glycaemic response following consumption of sugar water or starch-based food items ${ }^{(8-14)}$. For example, Torronen et $a l .{ }^{(13,14)}$ demonstrated that consuming polyphenol-rich berry nectars or berry purees with a high-carbohydrate food favourably modulated postprandial glycaemia in healthy adults by slowing glucose absorption and enhancing insulin and glucagon-like peptide-1 (GLP-1) secretion. However, an acknowledged limitation of those studies was a higher dietary fibre content and viscosity of the berry interventions relative to control, as both fibre and viscosity influence postprandial glycaemia and endocrine responses. In contrast, studies by CastroAcosta $^{(8,9)}$ observed a blunted glycaemic response when a starchy meal was provided with polyphenol-rich apple and blackcurrant extracts (i.e. devoid of fibre). With regard to the mechanisms of the glycaemic and glucoregulatory hormone modulation, in vitro and animal studies suggest that polyphenols, including those found in berries and in extracts of polyphenol-rich foods, can inhibit the carbohydrate digestive enzymes $\alpha$-amylase and $\alpha$-glucosidase, slow glucose absorption, modulate secretion of insulin and/or the incretin hormones glucose-dependent insulinotropic polypeptide (GIP) and GLP- $1^{(9,14,15)}$ and stimulate glucose uptake into insulin-sensitive tissues through increased activation of insulin receptors ${ }^{(7)}$. As such, polyphenol fortification of high-carbohydrate foods may help improve postprandial glycaemic control. This could have the added benefits of reducing free fatty acid release and oxidative stress ${ }^{(16)}$ and preventing increases in appetite that may result from rapid drops in blood glucose concentrations ${ }^{(7)}$.

Our group and others have previously reported that cranberry and black raspberry polyphenols inhibit $\alpha$-amylase and $\alpha$-glucosidase activities in vitro ${ }^{(17-19)}$. The primary objective of this study was to translate those findings to human metabolism by determining dose-response effects of fortifying a highcarbohydrate food with freeze-dried black raspberries or with cranberry extract on postprandial glycaemia (i.e. glucose incremental AUC with respect to baseline, AUC, 0-180 min) in healthy adults. Fortified and non-fortified cereal bars were created that were approximately matched for fibre, macronutrients and physicochemical characteristics. Secondary objectives were to determine the effects on postprandial glucoregulatory hormone responses, appetite and ex vivo oxidative stress. We hypothesised that polyphenol fortification would result in a dosedependent improvement in postprandial metabolic profiles and reduce appetite and ex vivo oxidative stress.

\section{Methods \\ Participants}

Participants were military and civilian personnel assigned to Natick Soldier Systems Center, Natick, MA. Twenty of the twenty-one participants who were enrolled and began the study completed data collection and were included in the data analyses. One participant was withdrawn from the study before consuming any of the cereal bars due to multiple failed catheter placements. Data collection occurred from January to November 2016 at the U.S. Army Research Institute of Environmental Medicine (USARIEM, Natick, MA). Each participant gave their written, informed consent after an oral explanation of the study. Men and women were included if they were 18-39 years; were generally healthy; had no history of liver disease, alcoholism, impaired glucose metabolism, thyroid disease, bleeding disorders, or gastrointestinal-related conditions that may impact glucose absorption; and had no allergy or aversion to any of the test foods. The study was approved by the Institutional Review Board, USARIEM. Investigators adhered to the policies for protection of human subjects as the prescribed DOD Instruction $3216 \cdot 02$, and the research was conducted in adherence to the provisions of 32 CFR Part 219. The Clinicaltrials.gov identifier is NCT02763020.

\section{Design}

This was a randomised, placebo-controlled, crossover trial conducted over five experimental sessions each separated by $\geq 5 \mathrm{~d}$ (9.5 (SD 5.2) d). Participants were assigned to experimental conditions using online software, Research Randomizer (www. randomizer.org). Flavour profile and colour of the bars provided some indication of the fruit contents; however, participants were unaware of which bars contained high $v$. low doses of the fruit ingredients. Study participants received written and verbal instructions to consume a low-polyphenol diet for two consecutive days before each session. Participants also consumed a provided dinner meeting approximately $1 / 3$ of their estimated weight maintenance energy requirements the evening before testing.

On test days, participants arrived following a $\geq 12 \mathrm{~h}$ overnight fast. Adherence to pre-trial dietary restrictions and consumption of the standardised dinner meal were verified using food records that were reviewed by research dietitians during each session. Following IV catheter placement, participants consumed one of five cereal bars in $\leq 15 \mathrm{~min}$. After bar consumption, the overall acceptability was rated on a Likert scale ranging from 1 (dislike extremely) to 9 (like extremely). Blood samples were collected and appetite was rated before and periodically for $180 \mathrm{~min}$ after bar consumption, to detect both the early and late postprandial responses of the outcome measures and to facilitate the ad libitum lunch test by providing a more realistic time frame between the 'breakfast' and lunch meals. During the $180 \mathrm{~min}$ postprandial period, participants remained seated and supervised and were not provided additional food or beverages other than $360 \mathrm{~g}$ of water. After $180 \mathrm{~min}$, energy intake was measured during an ad libitum lunch.

\section{Description of high-carbohydrate snack bars}

Five different fibre and macronutrient-matched high-carbohydrate cereal bars were tested (Table 1). The placebo bar contained no freeze-dried fruit or fruit extract. Two bars contained freeze-dried black raspberries (10\% (LOW-Rasp) or 20\% (HIGH-Rasp) total weight), and two bars contained cranberry extract (0.5\% (LOW-Cran) or $1.0 \%$ (HIGH-Cran) total weight). The base bar 
Table 1. Nutritional composition and acceptability of cereal bars* (Mean values and standard deviations)

\begin{tabular}{|c|c|c|c|c|c|}
\hline & \multirow[b]{2}{*}{ Reference } & \multicolumn{2}{|c|}{ Black raspberry } & \multicolumn{2}{|c|}{ Cranberry } \\
\hline & & Low† & High $\ddagger$ & Low§ & Highll \\
\hline Weight (g) & 124 & $122 \cdot 9$ & $121 \cdot 6$ & 124.9 & $125 \cdot 5$ \\
\hline Energy (kJ) & 1921 & 1918 & 1887 & 1928 & 1948 \\
\hline Carbohydrates (g) & 100 & $100 \cdot 7$ & 99 & $101 \cdot 7$ & $102 \cdot 2$ \\
\hline Total sugars $(\mathrm{g})$ & 45 & 45.5 & 44 & $45 \cdot 3$ & 45.4 \\
\hline Fructose $(\mathrm{g})$ & 3.9 & $5 \cdot 8$ & $4 \cdot 1$ & 4 & $4 \cdot 3$ \\
\hline Sucrose $(g)$ & $17 \cdot 3$ & $14 \cdot 4$ & $15 \cdot 2$ & $11 \cdot 7$ & $15 \cdot 7$ \\
\hline Glucose $(\mathrm{g})$ & 14.5 & $15 \cdot 9$ & $15 \cdot 8$ & 19.9 & $16 \cdot 3$ \\
\hline Maltose $(\mathrm{g})$ & $9 \cdot 3$ & 9.5 & 8.9 & $9 \cdot 7$ & $9 \cdot 2$ \\
\hline Total fibre (g) & $6 \cdot 4$ & 7.9 & $7 \cdot 1$ & $9 \cdot 1$ & 7.3 \\
\hline Soluble fibre $(\mathrm{g})$ & 0 & 0 & 0 & 0 & 0 \\
\hline Insoluble fibre $(\mathrm{g})$ & 6.4 & 7.9 & $7 \cdot 1$ & $9 \cdot 1$ & $7 \cdot 3$ \\
\hline Starch $(\mathrm{g}) \boldsymbol{\top}$ & 48.6 & $47 \cdot 3$ & 47.9 & $47 \cdot 3$ & 49.5 \\
\hline Carbohydrates (\% total energy) & 87 & 88 & 88 & 88 & 88 \\
\hline Fat $(g)$ & 3.7 & 3.7 & 3.4 & 3.5 & 3.5 \\
\hline Fat (\% total energy) & 7 & 7 & 7 & 7 & 7 \\
\hline Protein $(\mathrm{g})$ & $6 \cdot 2$ & 5.5 & $5 \cdot 9$ & $5 \cdot 7$ & $6 \cdot 1$ \\
\hline Protein (\% total energy) & 5 & 5 & 5 & 5 & 5 \\
\hline \multicolumn{6}{|l|}{ Overall acceptability } \\
\hline Mean & $7 \cdot 4$ & $6 \cdot 9$ & $6 \cdot 6^{\star *}$ & $5 \cdot 2^{\star \star}$ & $5 \cdot 8^{\star \star}$ \\
\hline SD & $1 \cdot 2$ & 1.5 & 1.5 & $2 \cdot 3$ & $1 \cdot 6$ \\
\hline
\end{tabular}

* All bars were chemically analysed for nutritional content (Covance Laboratories Inc.).

† Contains $10 \%$ freeze-dried black raspberry powder per total weight.

‡ Contains $20 \%$ freeze-dried black raspberry powder per total weight.

$\S$ Contains $0.5 \%$ cranberry extract per total weight.

II Contains $1 \%$ cranberry extract per total weight.

I Starch content calculated as total carbohydrates minus total fibre and total sugar.

${ }^{\star *}$ Marginal model with Bonferroni corrections. Significantly different from reference $P \leq 0.01$.

consisted of rice crisp cereal, marshmallows, butter and vanilla extract. The bar was loosely modelled after a Rice Krispie Treat (Kellogg Company), in an effort to promote palatability and to provoke marked glycaemia, which was necessary for testing the efficacy of polyphenol supplementation to moderate the glycaemic response.

Fructose powder, glucose powder or wheat bran was added to the bars, to approximately match sugar and fibre content between bars. The cranberry extract and raspberry powder were chosen based on their polyphenolic content and previous work by members of our group and others, suggesting that the polyphenol components (e.g. anthocyanins and proanthocyanidines) in these fruits effectively inhibited $\alpha$-amylase and glucoamylase activity in vitro ${ }^{(17-19)}$. The LOW-Rasp and HIGH-Rasp bars contained approximately 0.6 and $1.2 \mathrm{~g}$ of total polyphenols, respectively, based on gram weight of the bars (Table 1) and data indicating that black raspberries contain $0.98 \mathrm{~g}$ of polyphenols per $100 \mathrm{~g}$ of whole fruit ${ }^{(20)}$ (i.e. or approximately $5.1 \mathrm{~g}$ polyphenols per $100 \mathrm{~g}$ of freeze-dried black raspberry powder). The LOW-Cran and HIGH-Cran bars contained 0.3 and $0.6 \mathrm{~g}$ of polyphenols, respectively, based on gram weight of the bars (Table 1) and data indicating that the cranberry extract contains $45 \mathrm{~g}$ of polyphenols per $100 \mathrm{~g}$ of extract ${ }^{(21)}$. The polyphenols contained within HIGH-Rasp and LOW-Rasp were mostly anthocyanins, ellagitannins, ellagic acid and quercitin ${ }^{(20,22)}$, while the HIGH-Cran and LOW-Cran mainly consisted of flavanols (e.g. epicatechin), flavonols (e.g. quercetin) and phenolic acids (e.g. benzoic acid and chlorogenic acid), in addition to other polyphenolic compounds ${ }^{(21)}$. The highest dose of each fruit was based on the maximum dose that could be incorporated without compromising the organoleptic properties of the bars. The lower doses were included to assess the dose-response effects.

\section{Blood sampling}

An indwelling catheter was placed in the participants' forearm or antecubital space upon arrival to the testing site. Blood samples were taken after catheter placement and every $15 \mathrm{~min}$ for the first hour and every $30 \mathrm{~min}$ thereafter (up to $180 \mathrm{~min}$ ) following bar consumption. Whole blood was collected into serum tubes for measurement of glucose, insulin and C-peptide, and chilled EDTA tubes for measurement of GIP, GLP-1 and acylated ghrelin. EDTA tubes contained 4-(2-aminoethyl)-benzenesulfonyl fluoride, hydrochloride ( $100 \mathrm{~mm} ; 50 \mu \mathrm{l} / \mathrm{ml}$ whole blood $)$, dipeptidyl peptidase inhibitor IV $(10 \mu \mathrm{l} / \mathrm{ml}$ whole blood $)$ and aprotinin $(770 \mathrm{nmol} / \mathrm{ml}$ whole blood, equivalent to 500 $\mathrm{KIU} / \mathrm{ml}$ whole blood). Following serum and plasma separation, samples were stored at $-80^{\circ} \mathrm{C}$ until analysis.

Glucose was measured on a Siemens Dimension Xpand Plus clinical chemistry analyser, while insulin and C-peptide were measured on a Siemens Immulite 2000 immunoassay system. GIP, active GLP-1 and acylated ghrelin were measured using the Milliplex MAP human metabolic hormone panel (Millipore), according to the manufacturer instructions. Assay sensitivity was $0.6 \mathrm{pg} / \mathrm{ml}$ for GIP, $1.2 \mathrm{pg} / \mathrm{ml}$ for GLP-1 and $13 \mathrm{pg} / \mathrm{ml}$ for acylated ghrelin.

\section{Ex vivo $L D L$ resistance against $\mathrm{Cu}^{2+}$-induced oxidation}

Postprandial oxidative stress is regarded as a secondary response to postprandial hyperglycaemia and 
hypertriglyceridaemia ${ }^{(23)}$. While there are many markers for assessment of oxidative stress, LDL oxidation was selected as a biomarker because of the involvement of oxidised LDL in the development of atherosclerosis. For example, Natella et al. ${ }^{(24)}$ reported that postprandial LDL was more susceptible to metalcatalysed oxidation than the homologous baseline LDL after an ethanol meal. It was anticipated that postprandial hyperglycaemia may have the same impact on LDL susceptibility to oxidation as acute hyperglycaemia-induced oxidative stress in healthy people ${ }^{(25)}$.

Plasma was mixed with sucrose ( $0.6 \%$ final concentration), aliquoted and stored at $-80^{\circ} \mathrm{C}$. An ex vivo LDL oxidation assay was performed within 2 months of the sample collection. LDL $(1.019-1.063 \mathrm{~g} / \mathrm{ml})$ was collected from the frozen plasma according to Chung et al. ${ }^{(26)}$ using a Beckman NVT-90 rotor (or similar) in a Beckman L8-M centrifuge (or similar). After salt removal using a desalting PD-10 column (Bio-Rad), the concentration of LDL was determined using a bicinchoninic acid protein assay kit (Pierce; or similar). Ex vivo LDL oxidation induced by $\mathrm{Cu}^{2+}$ was performed according to the method described by Chen et al. ${ }^{(16)}$. Formation of conjugated dienes was monitored by absorbance at $234 \mathrm{~nm}$ at $37^{\circ} \mathrm{C}$ for $6 \mathrm{~h}$ using a Shimadzu UV1800 spectrophotometer equipped with a sixposition automated sample changer. The results of the assay are expressed as lag time, the intercept at the abscissa in the dienetime plot.

\section{Plasma flavonoids and phenolic acids}

Flavonoids (including the flavanols catechin and epicatechin and the flavonols quercetin, myricetin and isorhamnetin) and phenolic acids (including protocatechuic, phenylacetic, gentisic acid, benzoic acid, sinapic, caffeic, ferulic, vanillic and $p$-coumaric acids) in plasma were determined according to Chen et $a l{ }^{(16)}$, to provide insight into oxidative stress results. Briefly, plasma was incubated with vitamin C-EDTA and $\beta$-glucuronidase $/$ sulfatase at $37^{\circ} \mathrm{C}$ for $45 \mathrm{~min}$. Phenolic acids and flavonoids in the resulting mixture were extracted with acetonitrile, dried under purified $\mathrm{N}_{2}$ gas and reconstituted with mobile phase A for HPLC analysis using an ESA CoulArray System (ESA Inc.). Analyte separation was achieved using a Zorbax ODS C18 column $(4.6 \times 250 \mathrm{~mm}, 3.5 \mu \mathrm{m})$. Quantification of phenolic acids and flavonoids in unknown samples were calculated based on the standard curves constructed using authentic standards with adjustment for the internal standard (4'-hydroxy-3'-methoxyacetophenone).

\section{Appetite testing}

Two separate visual analogue scales were administered before each blood sample collection to measure the self-perceived appetite $^{(27)}$. Participants rated their levels of hunger and fullness by marking anywhere on a $10 \mathrm{~cm}$ scale anchored by phrases representing opposite extremes of a spectrum (e.g. 'not at all hungry' and 'extremely hungry').

An ad libitum lunch was served within $10 \mathrm{~min}$ after the final blood sample to provide an objective measure of appetite ${ }^{(28,29)}$. The meal consisted of Stouffer's lasagna ( $41 \%$ carbohydrate,
$36 \%$ fat, $23 \%$ protein) and $240 \mathrm{~g}$ water. Participants were served 1653 (SD 57) g and instructed to eat until 'comfortably full'. The amount of uneaten lasagna was weighed to calculate the energy intake.

\section{Statistical analysis}

Sample size estimates based on peak postprandial glucose concentrations, and using mean and variance data from Torronen et $a l .^{(13,14,30)}$ indicated that twenty participants would allow detection of a $0.9 \mathrm{mmol} / \mathrm{l}$ (approximately $15 \mathrm{mg} / \mathrm{dl}$ ) difference in peak glucose between trials with power $=0.8$ and $\alpha=0.01$ to account for multiple comparisons.

Statistical analyses were conducted using the IBM SPSS statistical package version 24.0 (IBM Inc.). Data were examined for outliers both quantitatively and graphically, and normal distribution of data was examined via the Shapiro-Wilk test. All data, except glucose, appetite ratings, energy intake, and LDL lag, were $\log _{10}$ transformed for analysis to normalise distributions. Values that were below the assay limits of detection (13\% of values for GLP-1, 2\% for ghrelin, $4 \%$ for insulin) were replaced with the lowest detectable limit for that assay before analysis.

Time to peak (i.e. for glucose, insulin, GLP-1, GIP, C-peptide) or nadir (i.e. for ghrelin) concentrations, change from baseline (time 0) to peak (i.e. for glucose, insulin, GLP-1, GIP, C-peptide, fullness) or nadir (i.e. for ghrelin, hunger) concentrations $\left(\Delta_{\max }\right)$, and incremental AUC with respect to baseline from 0 to 60 and 0 to 180 min were computed for all outcomes to standardise the results and used in the analyses to detect any differences between bars with regard to initial (AUC 0-60) and overall (AUC 0-180) postprandial responses. Analyses were run separately for the raspberry and cranberry interventions because the study objective was to assess dose-response effects within each intervention type and not to compare interventions. Data were analysed using marginal models to test for the main effects of treatment. Baseline (i.e. time $0 \mathrm{~min}$ ) values were entered as covariates in the models, and carry-over effects were assessed by including terms for treatment order and its interaction with treatment. These terms were removed from the model if not significant. When significant main effects of treatment were observed, all possible $t$ tests were conducted using the Bonferroni correction to adjust for multiple comparisons. The Kruskall-Wallis test was used to test for main effects of treatment on plasma flavonoid/phenolic acid content, because transformations did not normalise the data distribution. When significant main effects of treatment were observed, MannWhitney tests were conducted using Bonferroni corrections to adjust for multiple comparisons. Results are presented as means and standard deviations, unless otherwise noted. Two-tailed $P$ values $\leq 0.05$ were considered statistically significant, and $P$ values $\leq 0 \cdot 10$ were considered trends.

\section{Results}

\section{Participant characteristics}

Eighteen men and two women completed the study (age: 24 (sD 5) years; BMI: 26.8 (sD 3.5) kg/m²) (Fig. 1). Energy and 


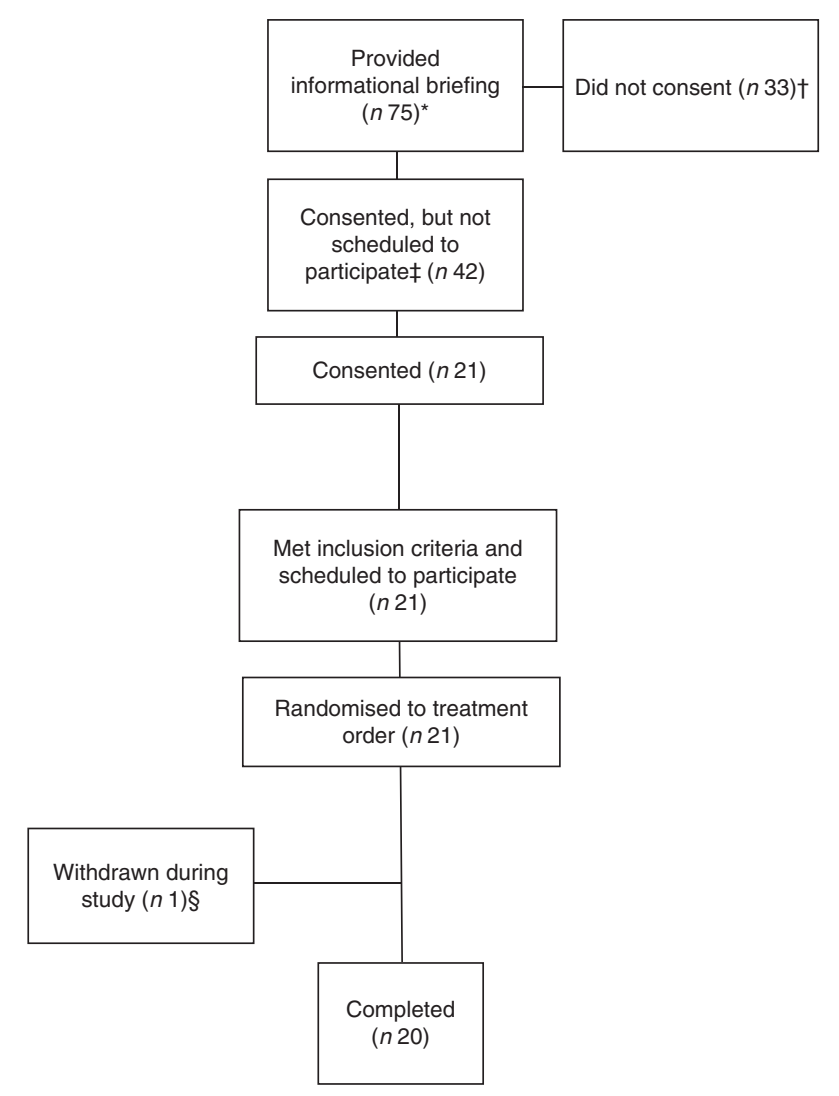

Fig. 1. Participant disposition. * Potential volunteers attended the briefing after seeing informational flyers or as part of their voluntary assignment in the Natick Soldier, Research, Development and Engineering Center's Human Volunteer Detachment (Natick, MA). † Two individuals verbally indicated that they did not meet inclusion criteria. $¥$ Not scheduled to participate due to scheduling conflicts. § Withdrawn due to multiple failed catheter attempts.

macronutrient intake in the $2 \mathrm{~d}$ before each trial, and in the evening meal before each trial, did not differ across trials $(P>0 \cdot 6)$ (online Supplementary Table S1). Body weight and fasting glucose and insulin concentrations did not differ across trials $(P>0 \cdot 3)$ (online Supplementary Table S2). Relative to the reference, the overall acceptability of the bars was rated lower for HIGH-Rasp and both cranberry bars (main effect of treatment, $P \leq 0 \cdot 02$; Table 1 )

\section{Glucose}

Within the raspberry treatments, there was a significant treatment effect on the overall glucose response $(P=0 \cdot 04)$, wherein a trend for a higher glucose $\mathrm{AUC}_{0-180 \mathrm{~min}}$ was observed during $\mathrm{HIGH}-$ Rasp $v$. LOW-Rasp $(P=0 \cdot 06$; Fig. $2(a))$. Glucose $\Delta_{\max }$, time to peak and $\mathrm{AUC}_{0-60 \text { min }}$ were not affected by treatment (Table 2 ).

The cranberry treatments had no effect on the postprandial glucose response (Table 2 and Fig. 2(b)).

\section{Insulin}

Within the raspberry treatments, there was a significant treatment effect on insulin $\Delta_{\max }(P=0.02)$, wherein the HIGH-Rasp response was lower relative to the reference (Table 2 and
Fig. 3). There were no effects of the raspberry treatments on time to peak insulin or insulin $\mathrm{AUC}_{0-60 \text { min }}$ or $\mathrm{AUC}_{0-180 \mathrm{~min}}$ (Table 2 and Fig. 2(c)).

The cranberry treatment had no effects on the postprandial insulin response (Table 2 and Fig. 2(d)).

\section{C-peptide}

Within the raspberry treatments (Fig. 2(e)), there was a significant treatment effect $(P=0 \cdot 01)$, wherein participants C-peptide $\mathrm{AUC}_{0-60}$ was lower during HIGH-Rasp (247 (sD 117) ng/dl) compared with the reference (279 (sD 243) ng/ $\mathrm{dl}$ ), but there was no effect on $\mathrm{AUC}_{0-180}$ or time to peak C-peptide (Table 2).

The cranberry treatments had no effects on the postprandial C-peptide response (Table 2 and Fig. 2(f)).

\section{Glucagon-like peptide-1}

Within the raspberry treatments, there was a trend for a treatment effect $(P=0.09)$, wherein GLP-1 $\Delta_{\max }$ trended towards being lower during HIGH-Rasp relative to the reference (Table 2 and Fig. 3(a)). No differences in time to peak GLP-1, $\mathrm{AUC}_{0-60 \text { min }}$ or $\mathrm{AUC}_{0-180 \text { min }}$ were observed (Fig. 3(a)).

Within the cranberry treatment, a carry-over effect was noted for GLP-1 AUC $0-180$ min. After removing the treatment sequence responsible for the carry-over effect, no differences across treatments were observed. No differences in time to peak GLP-1 or GLP-1 $\Delta_{\max }$ were observed (Table 2).

\section{Glucose-dependent insulinotropic polypeptide}

Within the raspberry treatments, there were significant treatment effects on the overall GIP response $(P<0 \cdot 05)$, wherein $\mathrm{AUC}_{0-60}$ and $\mathrm{AUC}_{0-180}$ for GIP were significantly lower following consumption of the HIGH-Rasp $v$. LOW-Rasp and/or reference bar (Fig. 3(c)). There was also a significant treatment effect $(P=0 \cdot 01)$, wherein GIP $\Delta_{\max }$ was lower after participants consumed HIGH-Rasp $v$. LOW-Rasp, but no effect on time to peak GIP was observed (Table 2).

The cranberry treatment had no effect on postprandial GIP response (Table 2 and Fig. 3(d)).

\section{Ghrelin}

The raspberry treatments had no effects on the postprandial acylated ghrelin response (Table 2 and Fig. 3(e)).

Within the cranberry treatment, there was a trend $(P=0.07)$ for a treatment effect, wherein the acylated ghrelin $\mathrm{AUC}_{0-180 \mathrm{~min}}$ trended towards being more negative (i.e. larger decrease) during HIGH-Cran relative to LOW-Cran $(P=0 \cdot 08)$. There was no effect of cranberry treatment on the maximal decrease in acylated ghrelin from baseline or time to nadir (Table 2 and Fig. 3(f)).

\section{Ex vivo $L D L$ resistance against $C u^{2+}$-induced oxidation}

There was no treatment effect when the raspberry bars were compared with one another and/or the reference bar (Fig. 4(a)). 
(a)

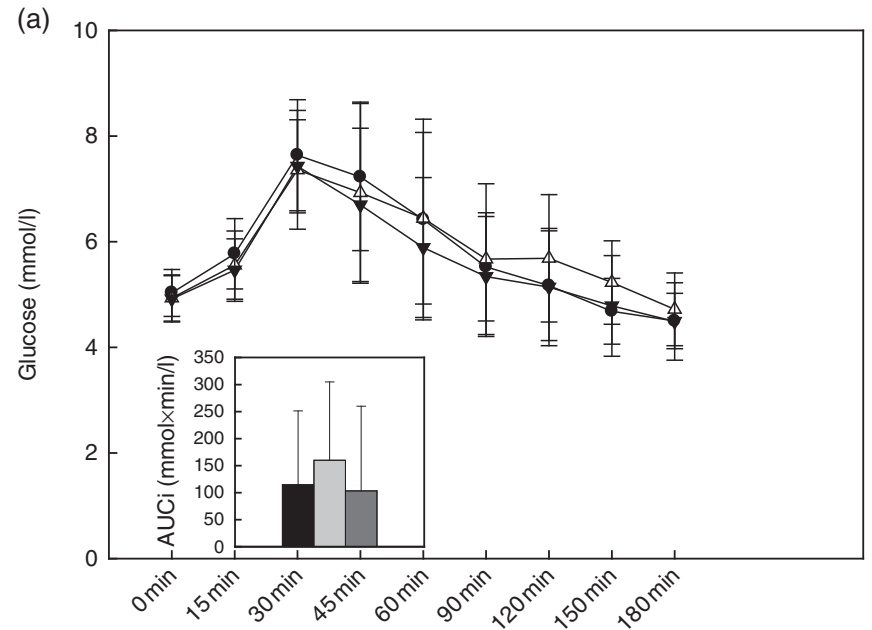

(c)
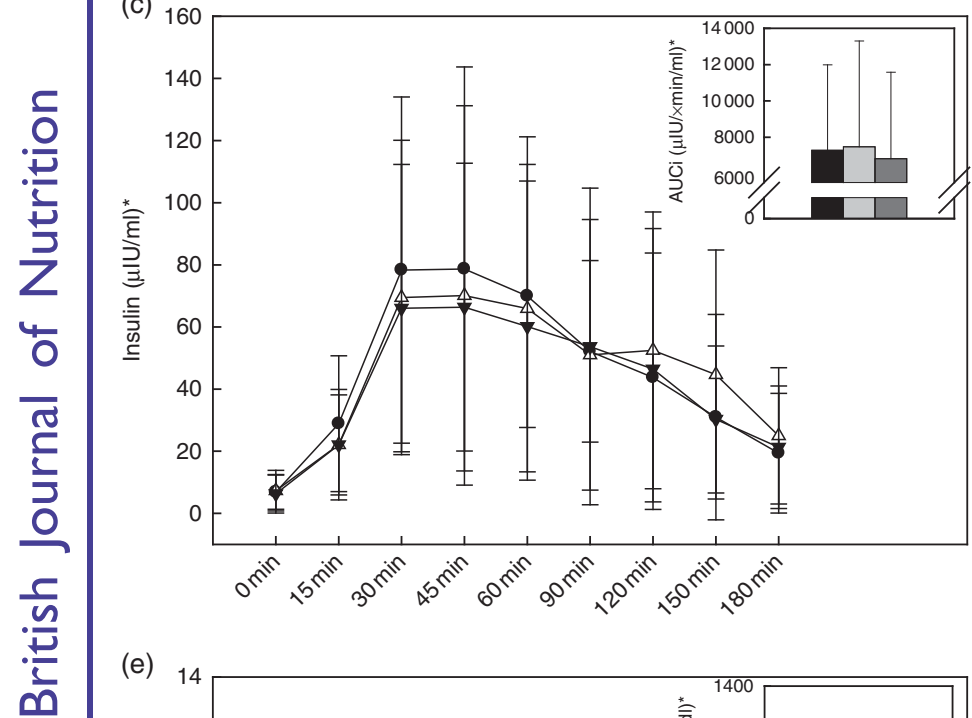

(e)

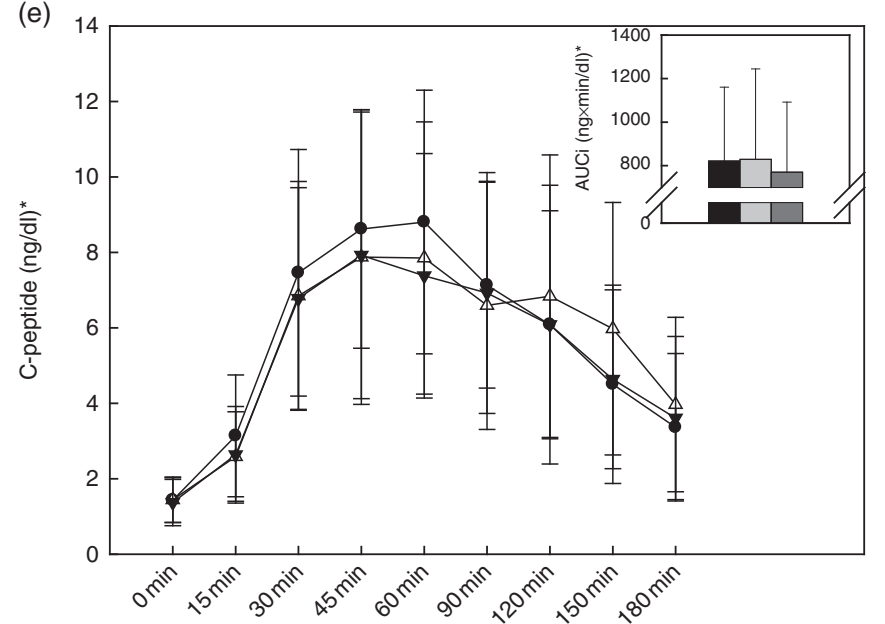

(b)

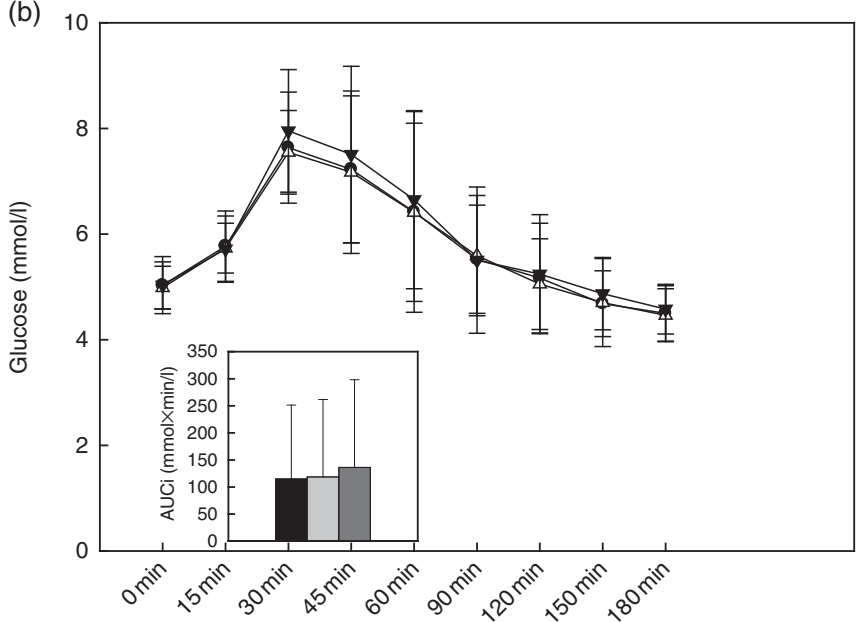

(d)

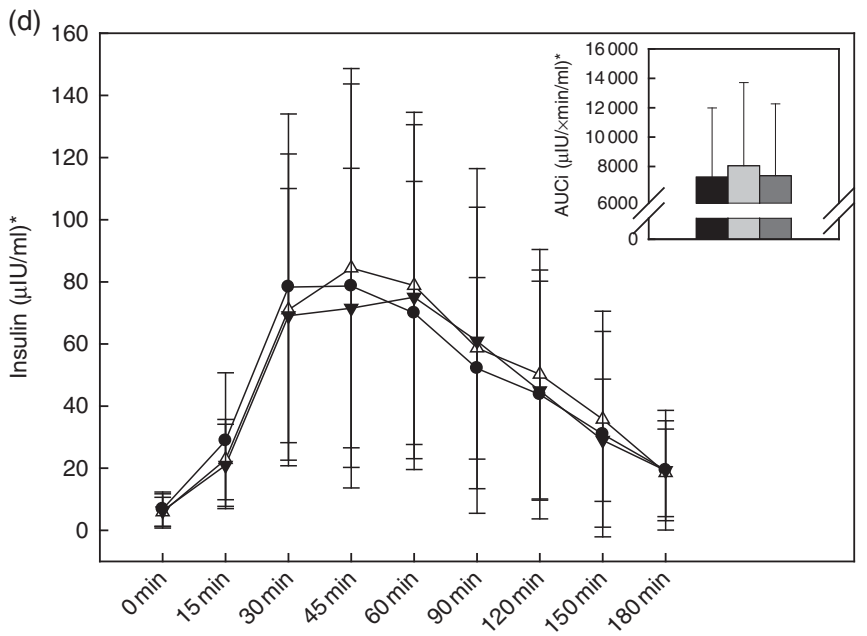

(f)

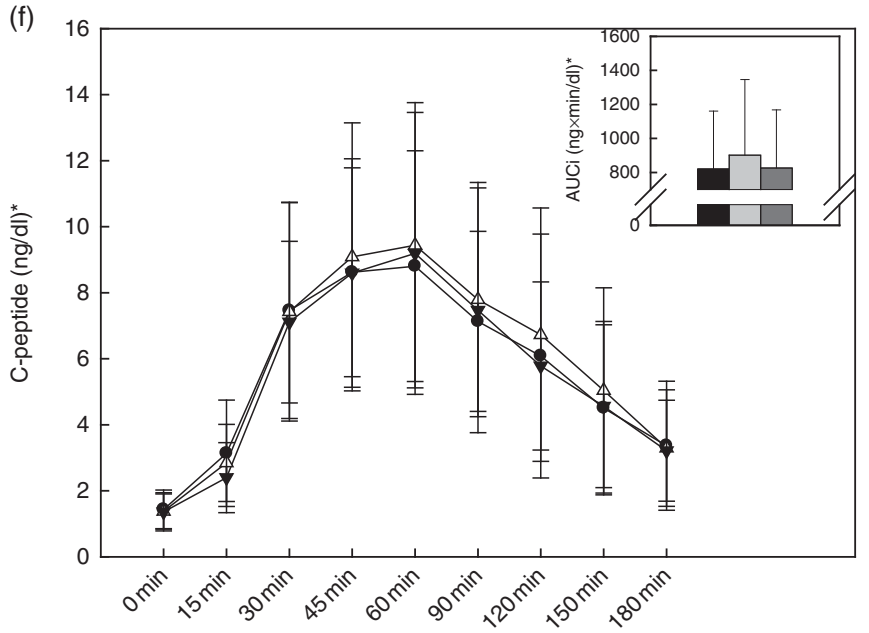

Fig. 2. Baseline and postprandial glucose (a, b), insulin (c, d) and C-peptide (e, f) concentrations. Insets show incremental $A U C$ (AUCi) $0-180$ min postprandial. Values are means, with standard deviations represented by vertical bars. There was a treatment effect on the glucose $\mathrm{AUC}_{0-180 \mathrm{~min}}$ response $(P=0.04)$ : trend for higher glucose following consumption of black raspberry high $v$. black raspberry low $(P=0.06)$. - and $\square$, Reference (a-f); $\square-$ and $\square$, black raspberry high (a, c, e), cranberry high (b, d, f); $\longrightarrow$ and $\square$, black raspberry low (a, c, e), cranberry low (b, d, f). * To convert insulin in $\mu$ lU/ml to pmol/l, multiply by 6.945 . To convert C-peptide in $\mathrm{ng} / \mathrm{dl}$ to $\mathrm{ng} / \mathrm{l}$, multiply by 10 . 
Table 2. Time to peak or nadir, and change from baseline to postprandial peak or nadir, blood concentrations of glucose, insulin, glucoregulatory and appetite-mediating hormones*

(Mean values and standard deviations)

\begin{tabular}{|c|c|c|c|c|c|c|c|c|c|c|}
\hline & & & \multicolumn{4}{|c|}{ Black raspberry } & \multicolumn{4}{|c|}{ Cranberry } \\
\hline & \multicolumn{2}{|c|}{ Reference } & \multicolumn{2}{|c|}{ Low† } & \multicolumn{2}{|c|}{ High $\ddagger$} & \multicolumn{2}{|c|}{ Low§ } & \multicolumn{2}{|c|}{ Highll } \\
\hline & Mean & SD & Mean & SD & Mean & SD & Mean & SD & Mean & SD \\
\hline Glucose $\Delta_{\max }(\mathrm{mmol} / \mathrm{l})$ & 2.9 & $1 \cdot 2$ & $2 \cdot 8$ & 0.9 & 2.9 & 0.9 & 2.9 & 1.0 & 3.3 & $1 \cdot 1$ \\
\hline Time to peak glucose (min) & 38 & 11 & 38 & 15 & 38 & 17 & 42 & 24 & 38 & 9 \\
\hline Insulin $\Delta_{\max }(\mu \mathrm{lU} / \mathrm{ml}) \boldsymbol{\Upsilon}$ & 90 & 64 & 78 & 55 & $75^{\star \star}$ & 53 & 84 & 58 & 82 & 52 \\
\hline Time to peak insulin (min) & 49 & 25 & 50 & 21 & 54 & 32 & 48 & 20 & 50 & 22 \\
\hline GLP-1 $\Delta_{\max }(\mathrm{pg} / \mathrm{ml})+\dagger$ & 25 & 15 & 21 & 14 & $18 \ddagger \ddagger$ & 13 & 27 & 25 & 19 & 14 \\
\hline Time to peak GLP-1 ( $\mathrm{min})$ & 41 & 40 & 33 & 26 & 26 & 14 & 45 & 40 & 38 & 26 \\
\hline GIP $\Delta_{\max }(\mathrm{pg} / \mathrm{ml}) \S \S$ & 201 & 103 & 211 & 82 & 162\|\| $\mid$ & 97 & 180 & 64 & 188 & 88 \\
\hline Time to peak GIP (min) & 78 & 31 & 95 & 42 & 71 & 36 & 60 & 24 & 74 & 33 \\
\hline C-peptide $\Delta_{\max }(\mathrm{mg} / \mathrm{dl})$ )ी & 8 & 3 & 8 & 3 & 8 & 3 & 9 & 4 & 9 & 4 \\
\hline Time to peak C-peptide ( $\mathrm{min}$ ) & 49 & 15 & 71 & 31 & 62 & 27 & 65 & 36 & 59 & 23 \\
\hline Ghrelin $\Delta_{\max }(\mathrm{pg} / \mathrm{ml})$ & -64 & 51 & -63 & 50 & -59 & 58 & -59 & 48 & -72 & 51 \\
\hline Time to nadir glucose ( $\mathrm{min}$ ) & 58 & 22 & 68 & 31 & 67 & 28 & 65 & 25 & 70 & 20 \\
\hline
\end{tabular}

$\Delta_{\text {max }}$, change from baseline to peak glucose, insulin, GLP-1, GIP, c-peptide concentrations or nadir acylated ghrelin concentrations; GLP-1, glucagon-like peptide-1; GIP, glucosedependent insulinotropic polypeptide.

* Data analysed using marginal models with Bonferroni corrections. $\Delta_{\max }$ analyses included fasting blood concentrations as a covariate.

† Contains $10 \%$ freeze-dried black raspberry powder per total weight.

$\ddagger$ Contains $20 \%$ freeze-dried black raspberry powder per total weight.

$\S$ Contains $0.5 \%$ cranberry extract per total weight.

II Contains $1 \%$ cranberry extract per total weight.

I Significant main effect of bar within raspberry treatment $(P=0.02)$. To convert insulin in $\mu \mathrm{lU} / \mathrm{ml}$ to pmol/l, multiply by 6.945 .

** $P=0.03$ v. reference.

†† Trend for main effect of bar within raspberry treatment $(P=0.09)$.

㧊 $P=0.09 \mathrm{v}$. reference.

$\S \S$ Significant main effect of bar within raspberry treatment $(P=0.01)$.

IIII $P=0.01$ v. LOW-Rasp.

ๆๆ To convert C-peptide in ng/dl to ng/l, multiply by 10 .

There were no treatment, time or treatment $x$ time interactions when the cranberry bars were compared with one another and/or the reference bar (Fig. 4(b)).

\section{Flavonoids and phenolic acids}

The raspberry treatment had no effects on plasma concentrations of measured flavonoids or phenolic acids (select phenolic acids shown in online Supplementary Figs. S1 and S2).

Within the cranberry treatment, there were significant treatment effects on the plasma concentrations of phenolic acids (i.e. gentisic, vanillic, caffeic, coumaric, ferulic and sinapic acids, $P<0.05)$ but not flavonoids. Most notably, post hoc testing indicated that gentisic acid was significantly higher after HIGH-Cran $v$. LOW-Cran and/or the reference bar; coumaric, ferulic and sinapic acids were significantly higher after consumption of HIGH-Cran and LOW-Cran compared with the reference bar; and vanillic acid and caffeic acid were significantly higher after LOW-Cran or HIGH-Cran, respectively, $v$. the reference bar $(P<0.02)$ (online Supplementary Figs. S1 and S2).

\section{Appetite}

Within the raspberry treatments, there was a trend for a main effect of treatment on ad libitum energy intake $(P=0 \cdot 10)$, but post hoc testing did not indicate significant differences between treatments. The raspberry treatments did not impact postprandial hunger or fullness ratings (Table 3).
Within the cranberry treatments, there was a trend for a main effect of treatment on fullness $\operatorname{AUC}_{0-180 \text { min }}(P=0 \cdot 06)$, with post hoc comparisons indicating a trend for lower fullness during HIGH-Cran relative to the reference bar $(P=0 \cdot 06)$. A trend for a main effect of treatment on the peak change in fullness $(P=0.08)$ was also observed, but post boc testing did not indicate significant differences between treatments. The cranberry treatments did not impact hunger ratings or ad libitum energy intake (Table 3).

\section{Discussion}

The main findings of this study were that fortifying a highcarbohydrate cereal bar with a high dose, but not a smaller dose, of freeze-dried raspberries blunted postprandial peak insulin and incretin hormone responses compared with an unfortified bar and tended to increase postprandial glucose AUC but not peak concentrations. Together, these findings suggest that fortifying a high-carbohydrate bar with a high dose, but not a smaller dose, of freeze-dried black raspberry powder attenuated postprandial insulinaemia and slowed glucose absorption. These results are supported by reduced GIP concentrations following consumption of the high-dose raspberry bar, as GIP is a sensitive marker of intestinal glucose uptake, and no differences in plasma glucose concentrations were observed in the $60 \mathrm{~min}$ postprandial period (when the predominant influence on blood glucose is absorption rate). These effects did not result in differences in appetite or ex vivo LDL resistance against oxidation, and 
(a)

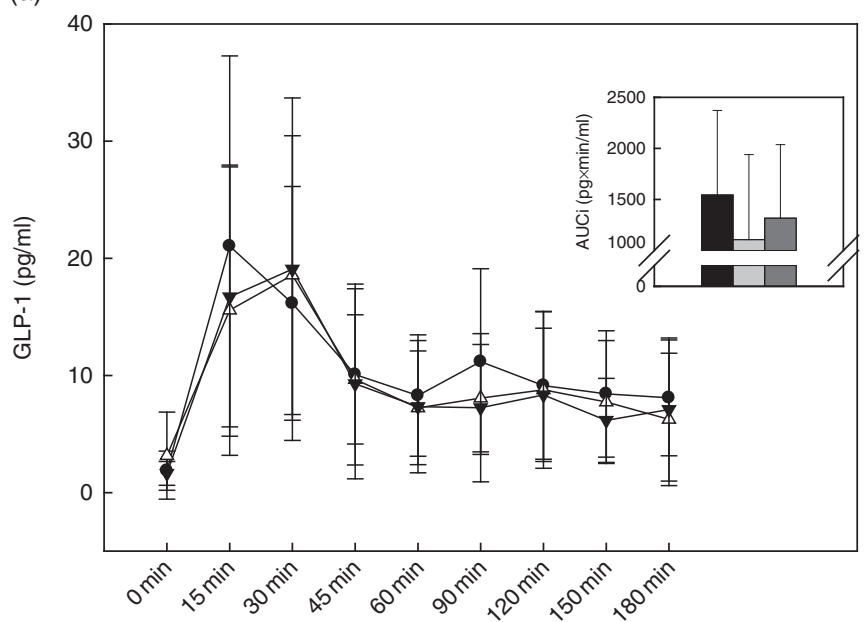

(c)
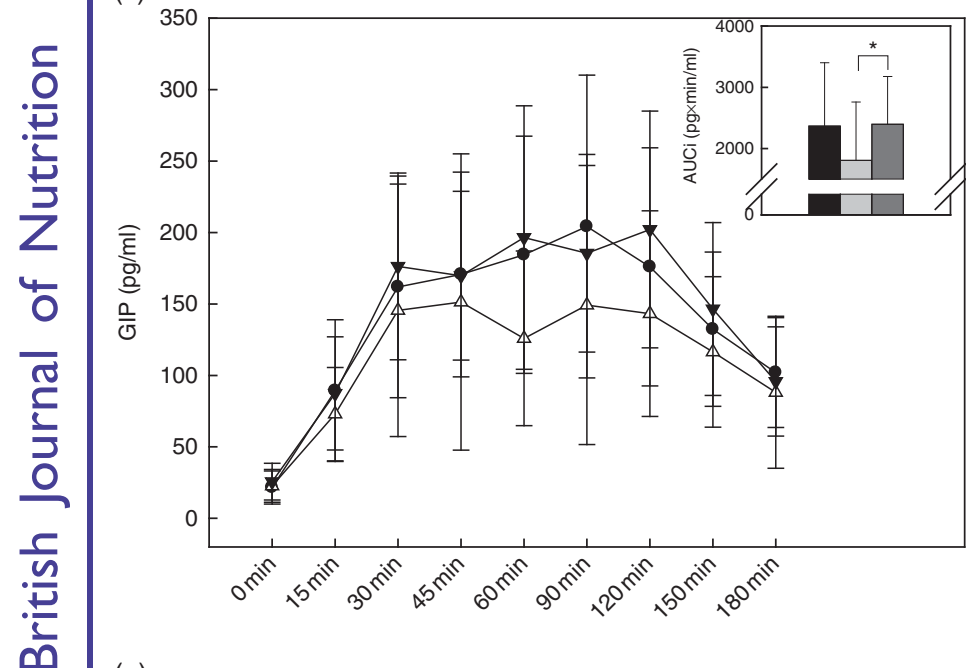

(e)

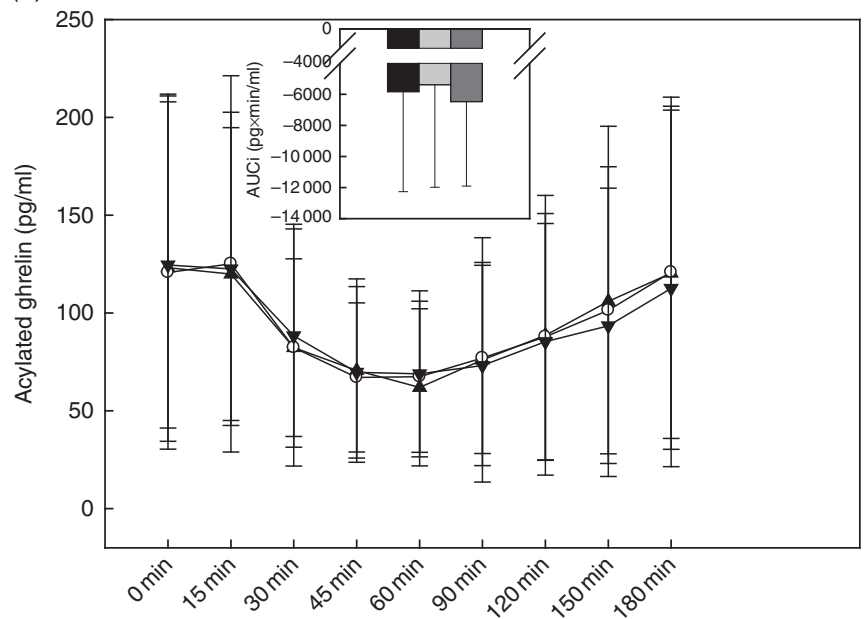

(b)

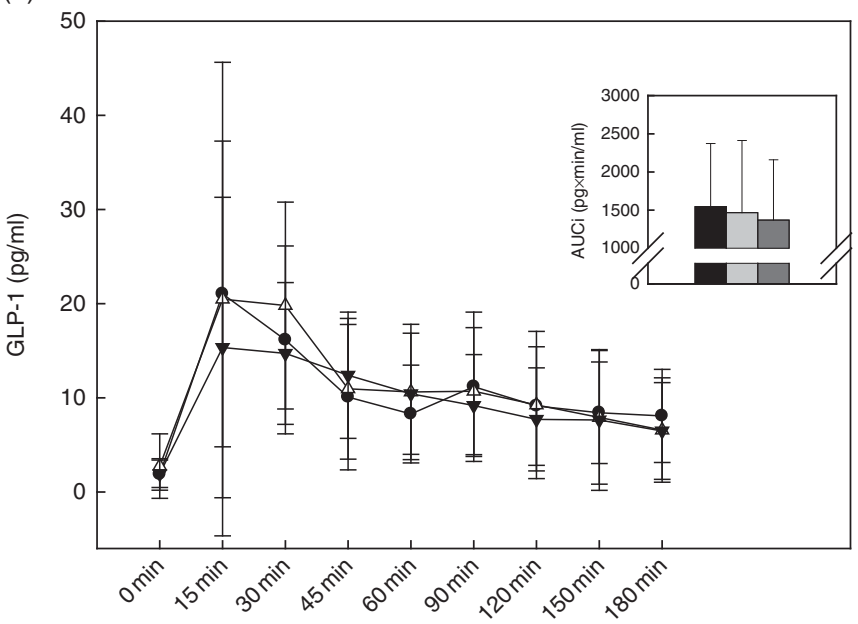

(d)

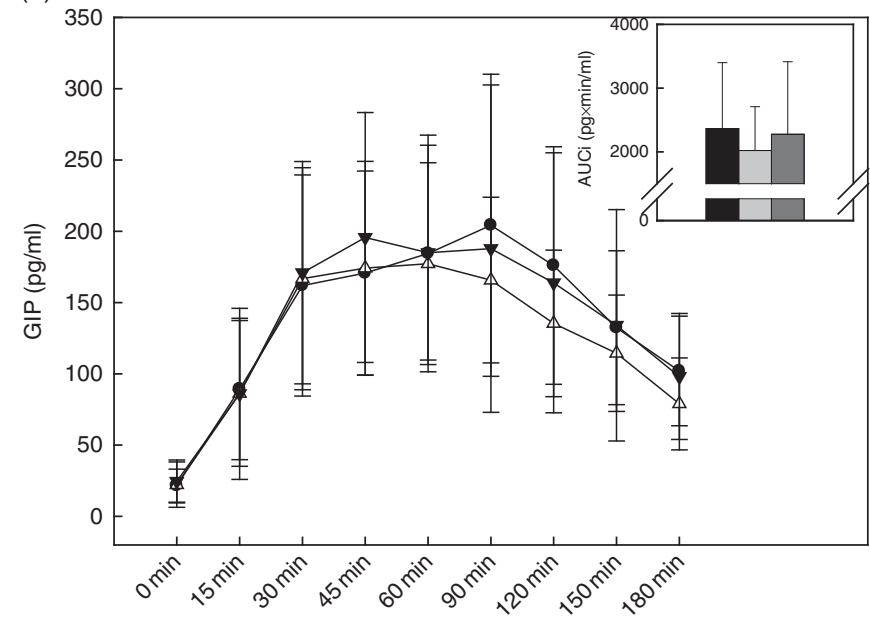

(f)

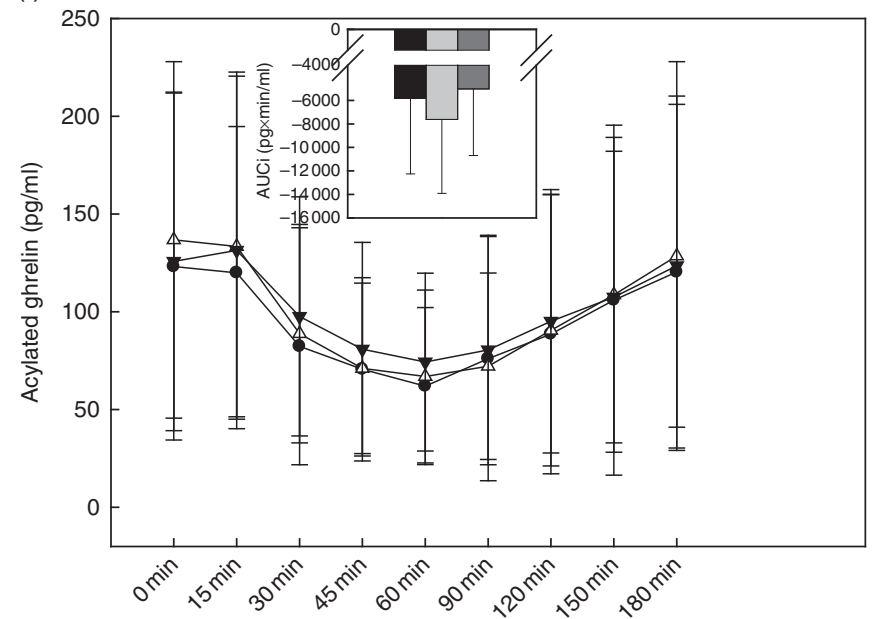

Fig. 3. Baseline and postprandial glucagon-like peptide-1 (GLP-1) (a, b), glucose-dependent insulinotropic polypeptide (GIP) (c, d) and acylated ghrelin (e, f) concentrations. Insets show incremental AUC (AUCi) 0-180 min postprandial. Values are means, with standard deviations represented by vertical bars. ${ }^{*}$ Significant difference between groups $(P<0.05)$. There was a treatment effect on GIP AUC ${ }_{0-180 m i n}$ response $(P=0.001)$ : GIP was lower following the consumption of black raspberry high $v$. reference $(P=0.014)$ and black raspberry low $(P=0.003)$. -- and $\square$, Reference $(a-f) ;-\square$ and $\square$, black raspberry high (a,c,e), cranberry high $(\mathrm{b}, \mathrm{d}, \mathrm{f}) ; \longrightarrow$ and $\square$, black raspberry low (a, c, e), cranberry low (b, d, f). 


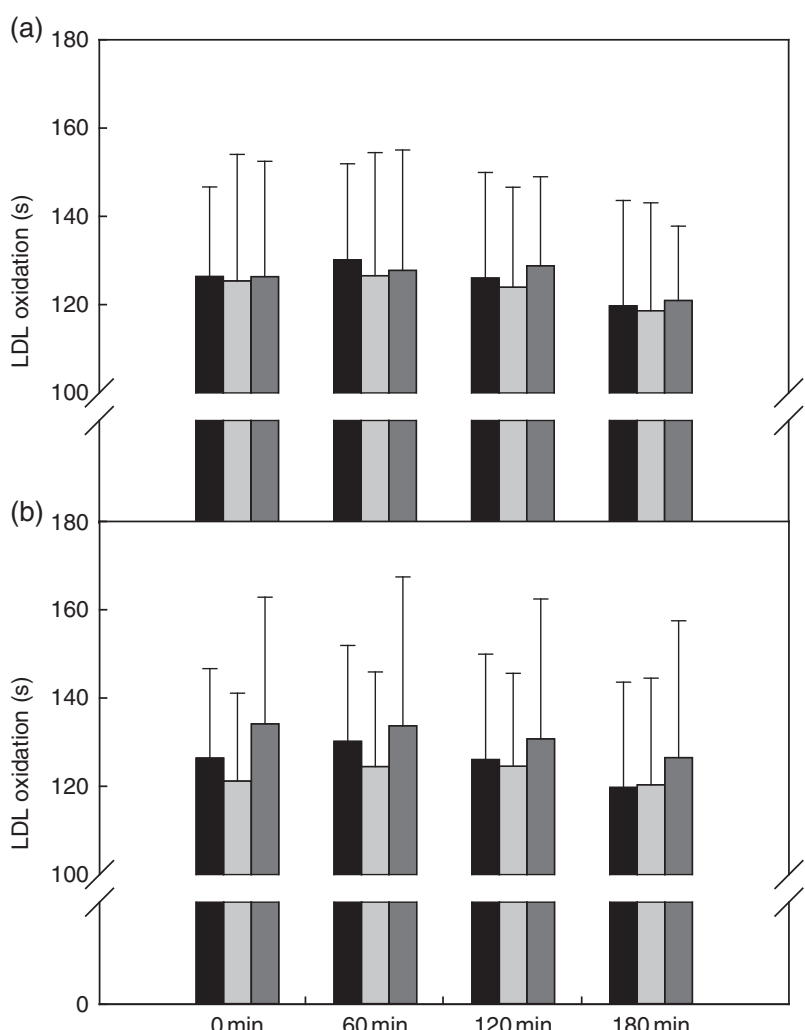

Fig. 4. Ex vivo LDL oxidation (lag time, $s$ ) of (a) reference $v$. black raspberryfortified bars and (b) reference $v$. cranberry-fortified bars. Values are means, with standard deviations represented by vertical bars. $\square$, Reference $(a, b) ; \square$, black raspberry high (a), cranberry high (b); $\square$, black raspberry low (a), cranberry low (b).

fortification with a polyphenol-rich cranberry extract did not elicit similar effects.

Our findings demonstrate that the high-dose raspberry bar favourably modulated postprandial glucose and glucoregulatory hormone responses. The $43 \%$ higher postprandial glucose AUC, but no difference in peak glucose concentrations, following consumption of the high-dose compared with the low-dose raspberry bar suggests delayed glucose absorption or uptake into peripheral tissues. The former response could reduce the postprandial insulin demand ${ }^{(7,31)}$, while the latter could be driven by lower postprandial insulin concentrations. Although diminished postprandial insulinaemia was not observed when comparing the high-dose with low-dose raspberry bar, the high-dose bar did show a modest blunting of postprandial insulin, C-peptide and glucoregulatory hormone responses when compared with the reference bar which is consistent with other studies ${ }^{(14,15)}$. The insulinaemic response to the high-dose black raspberry bar, compared with the reference, is consistent with the observation that C-peptide was lower in the $60 \mathrm{~min}$ following consumption of the high-dose black raspberry bar compared with the reference. C-peptide is not used by the liver and other organs, thus it is a more sensitive biomarker for endogenous insulin secretion compared with insulin itself due to unknown variability in tissue clearance of insulin ${ }^{(32)}$. In vitro studies demonstrating that polyphenols extracted from a variety of flavanol-rich foods inhibit digestive enzymes - specifically $\alpha$-amylase, $\alpha$-glycosidase and glucoamylase - during the breakdown of dietary carbohydrates into glucose ${ }^{(17)}$ provide a plausible mechanism. For example, recent in vitro studies demonstrate the inhibitory effects of different plants/extracts (e.g. grapeseed extract and African pear fruit) on $\alpha$-amylase and $\alpha$-glycosidase ${ }^{(11,33-36)}$. In addition, the raspberry powder used in the present study has exhibited similar effects in vitro, and these actions would be expected to inhibit starch digestion and slow glucose absorption ${ }^{(7,13,14,19)}$. Alternately, the blunted GIP and GLP-1 responses following the highdose fortification could underpin the lower postprandial C-peptide and insulin concentrations which could slow glucose uptake into peripheral tissues. Regardless of the mechanism, previous studies suggest that one possible advantage of slowed glucose absorption and/or uptake is improved physical and cognitive performance, especially during exercise ${ }^{(1-5)}$.

We acknowledge the inconsistency of not finding evidence for delayed glucose absorption when comparing the high-dose raspberry and reference bar despite the blunted insulin and glucoregulatory hormone responses. However, consistent with our results, three prior studies reported that the initial insulin response to a glucose beverage or starch-based food (i.e. bread) was attenuated by berries with little or no appreciable effect on glycaemic response ${ }^{(37-40)}$. These findings suggest an alternative mechanism whereby postprandial glucose metabolism may require less insulin when polyphenol-rich foods are consumed with high-carbohydrate foods, compared with high-carbohydrate foods alone ${ }^{(37,39-42)}$. Although the underpinning mechanisms have not been clearly defined, polyphenols may possibly act acutely to improve insulin sensitivity in peripheral tissue $7,31,39,40)$. We also acknowledge the inconsistency in the observation that the overall glucose response $\left(\mathrm{AUC}_{0-180 \mathrm{~min}}\right)$ was higher following consumption of HIGH-Rasp $v$. LOW-Rasp, in the absence of a higher overall insulin response. This suggests a lack of compensatory insulin secretion, possibly secondary to a lower overall GIP response, given that GIP is an incretin hormone. Indeed, inhibition of GIP has been observed in response to anthocyaninrich blackcurrants ${ }^{(8)}$. As suggested by others, this effect may be attributable to delayed glucose uptake in response to polyphenols as GIP is secreted from the proximal region of the small intestine, whereas glucose absorption may be occurring more distally because of the polyphenols ${ }^{(8,10)}$.

The observed effects of fortifying high-carbohydrate foods with polyphenol-rich foods on postprandial glycaemia were similar in magnitude (with regard to AUC) to a recent study that supplemented starch (i.e. white bread) with a mixture of polyphenol and fibre-rich foods (e.g. green tea powder, apple peel, blackberry, blackcurrant, and strawberry freeze-dried powders $)^{(11)}$ but less pronounced than those reported in other studies ${ }^{(13,14,30)}$. While the estimated amounts of total polyphenols were similar between this study and those of Torronen et al., it is possible that the type of polyphenols provided, as well as the fibre content and viscosity of the test meals, may be partially responsible for this discrepancy. For example, prior trials demonstrating a substantially altered glycaemic response used whole berries or berry purees consisting mainly of anthocyanins and proanthoyanidins ${ }^{(13,14,30)}$, whereas the present study used freeze-dried raspberry powder which contained mostly anthocyanins, ellagitannins, ellagic acid and 
Table 3. Appetite and ad libitum energy intake following consumption of fortified and reference cereal bars ${ }^{\star}$ (Mean values and standard deviations)

\begin{tabular}{|c|c|c|c|c|c|c|c|c|c|c|}
\hline & & & \multicolumn{4}{|c|}{ Black raspberry } & \multicolumn{4}{|c|}{ Cranberry } \\
\hline & \multicolumn{2}{|c|}{ Reference } & \multicolumn{2}{|c|}{ Low† } & \multicolumn{2}{|c|}{ High $\ddagger$} & \multicolumn{2}{|c|}{ Low§ } & \multicolumn{2}{|c|}{ Highll } \\
\hline & Mean & SD & Mean & SD & Mean & SD & Mean & SD & Mean & SD \\
\hline \multicolumn{11}{|l|}{$A U C_{0-180 \min }(\mathrm{cm} \mathrm{min})$} \\
\hline Hunger & -530 & 372 & -386 & 418 & -451 & 448 & -409 & 388 & -432 & 428 \\
\hline 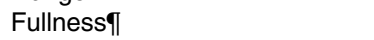 & 509 & 459 & 423 & 353 & 470 & 359 & 402 & 385 & $408^{\star *}$ & 379 \\
\hline \multicolumn{11}{|l|}{$\Delta_{\max }(\mathrm{cm})$} \\
\hline Hunger & -4.8 & $2 \cdot 7$ & -4.7 & $2 \cdot 7$ & $-4 \cdot 6$ & $2 \cdot 9$ & $-4 \cdot 6$ & $2 \cdot 1$ & -4.4 & 3. \\
\hline Fullness & 4.7 & $3 \cdot 2$ & 4.6 & $2 \cdot 3$ & 4.6 & $2 \cdot 8$ & 4.4 & $2 \cdot 3$ & $4 \cdot 1$ & $2 \cdot 5$ \\
\hline Ad libitum energy intake $(\mathrm{kJ}) \dagger^{\dagger}$ & 4925 & 1833 & 4573 & 1632 & 4891 & 2059 & 5130 & 1757 & 4975 & 1828 \\
\hline
\end{tabular}

$\Delta_{\max }$, change from baseline to peak fullness or the hunger nadir.

* Data analysed using marginal models with Bonferroni corrections. AUC and $\Delta_{\max }$ analyses included fasting hunger or fullness as a covariate.

t Contains $10 \%$ freeze-dried black raspberry powder per total weight.

$\ddagger$ Contains $20 \%$ freeze-dried black raspberry powder per total weight.

$\S$ Contains $0.5 \%$ cranberry extract per total weight.

II Contains $1 \%$ cranberry extract per total weight.

II Trend for main effect of bar within cranberry treatment $(P \leq 0.08)$

** $P=0.06 \mathrm{v}$. reference.

$\dagger \dagger$ Trend for main effect of bar within raspberry treatment $(P=0 \cdot 10)$. No significant post hoc differences.

quercitin. Further, the polyphenol-rich foods used in those studies contained more soluble fibre and were more viscous than their control foods. Authors of those studies asserted that the modified glucose response they observed was not solely attributed to differences in the soluble fibre content of the berry meals (up to $1.5 \mathrm{~g}$ ); however, it may partially explain their results since soluble fibre increases viscosity and mitigates postprandial glycaemic response to high-carbohydrate foods ${ }^{(43)}$. In contrast, the polyphenol-rich and reference bars used in the present study contained no soluble fibre and did not differ in viscosity. Indeed similar to the present study, Castro$\operatorname{Acosta}^{(8,9)}$ removed fibre as a potential confounding variable (i.e. by testing apple and blackcurrant extracts) and observed that the fruit extract modulated the glycaemic response to fruit juice and white bread with apricot jam. Differential findings between the aforementioned and present study may be attributable to the type of fruits, and their polyphenolic constituents, that were tested.

Contrary to our hypothesis, polyphenol fortification did not suppress appetite and did not dose dependently potentiate postprandial increases in GLP-1 or postprandial decreases in acylated ghrelin, both appetite-mediating hormones. Although relatively few studies have evaluated the acute effects of polyphenol-rich foods on appetite and associated hormones ${ }^{(44)}$, several have reported that consuming polyphenol-rich foods may alter appetite-mediating hormones in a direction that would be expected to suppress appetite. For example, in separate studies, adding a polyphenol-rich berry puree to sugar water potentiated postprandial increases in the appetitesuppressing hormone GLP-1 ${ }^{(14)}$, while adding polyphenol-rich soluble carob fibre to a liquid meal potentiated the postprandial suppression of the appetite-stimulating hormone acylated ghrelin $^{(45)}$. In vitro and animal studies suggest that certain polyphenols may directly modulate GLP-1 and ghrelin secretion and interact with hormones known to influence their biological activity $^{(44)}$. However, the effects of polyphenols on GLP-1 and acylated ghrelin appear to vary by polyphenol type ${ }^{(46,47)}$ and the food matrix in which polyphenols are consumed ${ }^{(45,48,49)}$. Importantly, studies demonstrating effects of polyphenol-rich foods on GLP-1 and/or ghrelin in a direction that would be expected to suppress appetite could not separate effects of polyphenols from the fibre and viscosity of the test meals ${ }^{(14,45)}$, which are both factors also thought to influence appetitemediating hormone responses ${ }^{(50)}$. In addition, a recent study found no acute effect of consuming $0.5-1.5 \mathrm{~g}$ of polyphenolrich grape seed extract on appetite ${ }^{(51)}$, despite the extract having been shown to inhibit $\alpha$-amylase and $\alpha$-glucosidase in vitro ${ }^{(52)}$, similar to the polyphenols used in the present study. Collectively, these findings do not support an appetite suppressing effect of berry polyphenols within the doses studied when fibre intake and viscosity are matched across fortified and non-fortified food products. Nonetheless, an impact of polyphenols on appetite is biologically plausible, given the evidence for roles of various polyphenols in modulating glucose metabolism and the concentrations of appetite-regulating neuropeptides and enteroendocrine hormones ${ }^{(7,44,46,53)}$. As such, these results may not pertain to other polyphenol forms or sources administered in different doses or other food matrices.

Findings from the present study indicate that neither of the fruit ingredients, in the doses provided, enhanced the ex vivo resistance of LDL lipids to oxidation following consumption of a high-carbohydrate snack bar. This is not surprising, given there was little effect of the reference bar on LDL oxidation. Postprandial oxidative stress, as a sub-form of nutritional oxidative stress, ensues from sustained postprandial hyperlipidaemia and/or hyperglycaemia and is associated with a higher risk of cardiometabolic diseases ${ }^{(54)}$. Even though absorbed polyphenols, including flavonoids and phenolic acids, may confer anti-oxidative protection to LDL against oxidation during the postprandial state, we speculated that the protection of LDL against oxidation might be mainly attributed to diminished postprandial glucose response derived from lower glucose absorption in the gastrointestinal tract. Thus, the interpretation of LDL oxidation data cannot solely focus on polyphenol 
bioavailability. In addition, this study only measured one biomarker to evaluate the effect of antioxidants within cranberry and black raspberry on oxidative stress, while a complete assessment of oxidative stress status from antioxidant interventions requires application of an array of biomarkers, including antioxidants (e.g. enzymes and small molecular antioxidants) and free radical-derived oxidised products. Altogether, future studies should consider assessing the effect of fortifying a high-fat food item with raspberry or cranberry polyphenols on LDL oxidation, using ex vivo or more robust in vivo methods, or in populations with increased susceptibility to oxidative stress such as the obese and elderly.

The raspberry and cranberry treatments produced differential effects on plasma concentrations of flavonoids and phenolic acids. Specifically, the raspberry treatment had no effect on circulating flavonoids or phenolic acid concentrations, whereas the cranberry treatment increased postprandial concentrations of several phenolic acids. These differences are probably attributable to the type of polyphenolic constituents within the berries and the methods of detection used in the present study. For example, anthocyanins are a main polyphenolic constituent of cranberries and black raspberries which are not quantified using the method described herein. Further, the bioavailability of polyphenols is also dependent on a number of factors that may have limited their detection in plasma, for example, chemical structure of the food matrix, intestinal absorption, interaction with gut microbiota and inter-subject differences in physiological, genetic and biochemical conditions ${ }^{(55)}$

\section{Limitations}

While the cereal bars were formulated to contain similar sugar and fibre composition, post-production chemical analysis revealed slight differences in fructose, sucrose and insoluble fibre content between the bars. However, these discrepancies are minor and unlikely to explain the differences we observed in glycaemic, insulinaemic and glucoregulatory hormone responses between the bars. Further, while polyphenol types within the bars were not measured, this data is reported in Phenol-Explorer ${ }^{(56)}$ by Wada \& Ou, and a detailed characterisation of the cranberry extract was published by Martín et al. In addition, the cyclical reproductive hormones for the two female participants were not considered, and the study was not powered to assess the differences between male and female. Lastly, we did not measure plasma anthocyanins due to cost constraints and instead chose to focus on flavonoids and phenolic acids, since the assay allowed measurement of more polyphenolic compounds. Despite these limitations, this was a comprehensive, highly controlled study examining the glycaemic, insulinaemic, glucoregulatory hormone and ex vivo oxidative stress responses following consumption of polyphenol-fortified, high-carbohydrate cereal bars.

\section{Conclusion}

Fortification with a high dose of freeze-dried black raspberries blunted postprandial peak insulin and incretin hormone responses compared with the reference bar and tended to increase postprandial glycaemia compared with the low-dose raspberry bar. Together, these findings suggest that fortifying a high-carbohydrate bar with approximately $25 \mathrm{~g}$ raspberry powder, which contained approximately $1.2 \mathrm{~g}$ of total polyphenols, modulated postprandial glycaemia and insulinaemia in a dosedependent manner, thus maintaining glucose availability in the postprandial period. Further research is warranted to determine whether this response has practical benefits in certain scenarios, for example, when the food item is consumed before endurance exercise or to maximise cognitive performance during the later postprandial period. However, these glycaemic effects did not translate into meaningful changes in appetite or ex vivo oxidation of LDL within the immediate postprandial period at the dose consumed and/or with the method used to assess appetite.

\section{Acknowledgements}

The authors would like to thank the study participants and technical support personnel within USARIEM's Military Nutrition Division, with whom this study would not have been possible.

The present study was funded by U.S. Army Medical Research and Materiel Command and Natick Soldier Research, Development and Engineering Center. This research was supported in part by an appointment to the Postgraduate Research Participation Program at the USARIEM administered by the Oak Ridge Institute for Science and Education through an interagency agreement between the United States Department of Energy and USARIEM.

The authors' contributions are as follows: T. J. S. designed the study, collected data, conducted analyses and drafted the manuscript; J. P. K. contributed to study design, collected data, conducted analyses, drafted portions of the manuscript related to satiety and revised the article for important intellectual content. M. A. W. and C. C. W. collected data and assisted in data analyses; C. C. W. also assisted in drafting the manuscript. A. B. and N. F. F. conceived of the study, assisted in study design and revised the article for important intellectual content; C.-Y. O. C. assessed LDL oxidation and plasma polyphenolic concentrations and revised the article for important intellectual content; S. J. M. contributed to study design and revised the article for important intellectual content. All authors reviewed, edited and approved the final manuscript.

The opinions or assertions contained herein are the private views of the authors and are not to be construed as official or reflecting the views of the US Army or the Department of Defense. Any citations of commercial organisations and trade names in this report do not constitute an official Department of the Army endorsement of approval of the products or services of these organisations. Study funded by the US Army Medical Research and Materiel Command.

The authors have no financial or personal conflicts of interest to declare.

\section{Supplementary material}

For supplementary material/s referred to in this article, please visit https://doi.org/10.1017/S0007114519000394 


\section{References}

1. Kirwan JP, Cyr-Campbell D, Campbell WW, et al. (2001) Effects of moderate and high glycemic index meals on metabolism and exercise performance. Metabolism 50, 849-855.

2. Kirwan JP, O'Gorman D \& Evans WJ (1998) A moderate glycemic meal before endurance exercise can enhance performance. J Appl Physiol 84, 53-59.

3. Kirwan JP, O'Gorman DJ, Cyr-Campbell D, et al. (2001) Effects of a moderate glycemic meal on exercise duration and substrate utilization. Med Sci Sports Exerc 33, 1517-1523.

4. Nilsson A, Radeborg K \& Bjorck I (2009) Effects of differences in postprandial glycaemia on cognitive functions in healthy middle-aged subjects. Eur J Clin Nutr 63, 113-120.

5. Nilsson A, Radeborg K \& Bjorck I (2012) Effects on cognitive performance of modulating the postprandial blood glucose profile at breakfast. Eur J Clin Nutr 66, 1039-1043.

6. Ludwig DS (2002) The glycemic index: physiological mechanisms relating to obesity, diabetes, and cardiovascular disease. JAMA 287, 2414-2423.

7. Hanhineva K, Torronen R, Bondia-Pons I, et al. (2010) Impact of dietary polyphenols on carbohydrate metabolism. Int J Mol Sci 11, 1365-1402.

8. Castro-Acosta ML, Smith L, Miller RJ, et al. (2016) Drinks containing anthocyanin-rich blackcurrant extract decrease postprandial blood glucose, insulin and incretin concentrations. J Nutr Biochem 38, 154-161.

9. Castro-Acosta ML, Stone SG, Mok JE, et al. (2017) Apple and blackcurrant polyphenol-rich drinks decrease postprandial glucose, insulin and incretin response to a high-carbohydrate meal in healthy men and women. J Nutr Biochem 49, 53-62.

10. Johnston KL, Clifford MN \& Morgan LM (2002) Possible role for apple juice phenolic compounds in the acute modification of glucose tolerance and gastrointestinal hormone secretion in humans. J Sci Food Agric 82, 1800-1805.

11. Nyambe-Silavwe H \& Williamson G (2016) Polyphenol- and fibre-rich dried fruits with green tea attenuate starch-derived postprandial blood glucose and insulin: a randomised, controlled, single-blind, cross-over intervention. Br J Nutr 116, 443-450.

12. Schulze C, Bangert A, Kottra G, et al. (2014) Inhibition of the intestinal sodium-coupled glucose transporter 1 (SGLT1) by extracts and polyphenols from apple reduces postprandial blood glucose levels in mice and humans. Mol Nutr Food Res 58, 1795-1808.

13. Torronen R, Kolehmainen M, Sarkkinen E, et al. (2012) Postprandial glucose, insulin, and free fatty acid responses to sucrose consumed with blackcurrants and lingonberries in healthy women. Am J Clin Nutr 96, 527-533.

14. Torronen R, Sarkkinen E, Niskanen T, et al. (2012) Postprandial glucose, insulin and glucagon-like peptide 1 responses to sucrose ingested with berries in healthy subjects. Br J Nutr 107, 1445-1451.

15. Williamson G (2013) Possible effects of dietary polyphenols on sugar absorption and digestion. Mol Nutr Food Res 57, $48-57$.

16. Chen CY, Milbury PE, Lapsley K, et al. (2005) Flavonoids from almond skins are bioavailable and act synergistically with vitamins $\mathrm{C}$ and $\mathrm{E}$ to enhance hamster and human LDL resistance to oxidation. J Nutr 135, 1366-1373.

17. Barrett A, Ndou T, Hughey CA, et al. (2013) Inhibition of alpha-amylase and glucoamylase by tannins extracted from cocoa, pomegranates, cranberries, and grapes. J Agric Food Chem 61, 1477-1486.

18. Martín MA, Ramos S, Mateos R, et al. (2015) Chemical characterization and chemo-protective activity of cranberry phenolic powders in a model cell culture. Response of the antioxidant defenses and regulation of signaling pathways. Food Res Int 71, 68-82.

19. Barrett AHFN, Fortier JS \& Apostolidis E (2015) Glucosidase Inbibition Effectiveness of Various Fruit Extracts and Their Acceptance in Performance Bar Products. Institute of Food Technologists Annual Meeting.

20. Wada L \& Ou B (2002) Antioxidant activity and phenolic content of Oregon caneberries. I Agric Food Chem 50 , 3495-3500.

21. Martín MA, Ramos S, Mateos R, et al. (2015) Chemical characterization and chemo-protective activity of cranberry phenolic powders in a model cell culture. Response of the antioxidant defenses and regulation of signaling pathways. Food Res Int 71, 68-82.

22. Gu J, Ahn-Jarvis JH, Riedl KM, et al. (2014) Characterization of black raspberry functional food products for cancer prevention human clinical trials. J Agric Food Chem 62, 3997-4006

23. Ceriello A \& Genovese S (2016) Atherogenicity of postprandial hyperglycemia and lipotoxicity. Rev Endocr Metab Disord 17, 111-116.

24. Natella F, Ghiselli A, Guidi A, et al. (2001) Red wine mitigates the postprandial increase of LDL susceptibility to oxidation. Free Radic Biol Med 30, 1036-1044.

25. Marfella R, Quagliaro L, Nappo F, et al. (2001) Acute hyperglycemia induces an oxidative stress in healthy subjects. J Clin Invest 108, 635-636.

26. Chung BH, Wilkinson T, Geer JC, et al. (1980) Preparative and quantitative isolation of plasma lipoproteins: rapid, single discontinuous density gradient ultracentrifugation in a vertical rotor. J Lipid Res 21, 284-291.

27. Blundell J, de Graaf C, Hulshof T, et al. (2010) Appetite control: methodological aspects of the evaluation of foods. Obes Rev 11, 251-270.

28. Karl JP, Young AJ \& Montain SJ (2011) Eating rate during a fixed-portion meal does not affect postprandial appetite and gut peptides or energy intake during a subsequent meal. Physiol Behav 102, 524-531.

29. O'Connor KL, Scisco JL, Smith TJ, et al. (2016) Altered appetite-mediating hormone concentrations precede compensatory overeating after severe, short-term energy deprivation in healthy adults. $J$ Nutr 146, 209-217.

30. Torronen R, Sarkkinen E, Tapola N, et al. (2010) Berries modify the postprandial plasma glucose response to sucrose in healthy subjects. Br J Nutr 103, 1094-1097.

31. Kim DJ, Jeong YJ, Kwon JH, et al. (2008) Beneficial effect of chungkukjang on regulating blood glucose and pancreatic beta-cell functions in C75BL/KsJ-db/db mice. J Med Food 11, 215-223.

32. Saisho Y (2016) Postprandial C-peptide to glucose ratio as a marker of $\beta$ cell function: implication for the management of type 2 diabetes. Int J Mol Sci 17, 744-752.

33. Miao M, Jiang H, Jiang B, et al. (2014) Phytonutrients for controlling starch digestion: evaluation of grape skin extract. Food Chem 145, 205-211.

34. Oboh G, Ademosun A, Olasehinde T, et al. (2015) Effect of processing methods on the antioxidant properties and inhibition of $\alpha$-amylase and $\alpha$-glucosidase by African pear (Dacryodes edulis) fruit. Nutrafoods 14, 19-26.

35. Sarikurkcu C (2015) An alternative antioxidative and enzyme inhibitory agent from Turkey: Robinia pseudoacacia L. Ind Crops Prod 78, 110-115.

36. Tong WY, Wang H, Waisundara VY, et al. (2014) Inhibiting enzymatic starch digestion by hydrolyzable tannins isolated from Eugenia jambolana. LWT - Food Sci Technol 59, 389-395. 
37. Torronen R, Kolehmainen M, Sarkkinen E, et al. (2013) Berries reduce postprandial insulin responses to wheat and rye breads in healthy women. J Nutr 143, 430-436.

38. Clegg ME, Pratt M, Meade CM, et al. (2011) The addition of raspberries and blueberries to a starch-based food does not alter the glycaemic response. Br J Nutr 106, 335-338.

39. Coe S \& Ryan L (2016) White bread enriched with polyphenol extracts shows no effect on glycemic response or satiety, yet may increase postprandial insulin economy in healthy participants. Nutr Res 36, 193-200.

40. Xu J, Jönsson T, Plaza M, et al. (2018) Probiotic fruit beverages with different polyphenol profiles attenuated early insulin response. Nutr J 17, 34-43.

41. Leinonen K, Liukkonen K, Poutanen K, et al. (1999) Rye bread decreases postprandial insulin response but does not alter glucose response in healthy Finnish subjects. Eur J Clin Nutr 53, 262-267.

42. Juntunen KS, Laaksonen DE, Autio K, et al. (2003) Structural differences between rye and wheat breads but not total fiber content may explain the lower postprandial insulin response to rye bread. Am J Clin Nutr 78, 957-964.

43. Wood PJ (2007) Cereal $\beta$-glucans in diet and health. $J$ Cereal Sci 46, 230-238.

44. Pinent M, Blay M, Serrano J, et al. (2017) Effects of flavanols on the enteroendocrine system: repercussions on food intake. Crit Rev Food Sci Nutr 57, 326-334.

45. Gruendel S, Garcia AL, Otto B, et al. (2006) Carob pulp preparation rich in insoluble dietary fiber and polyphenols enhances lipid oxidation and lowers postprandial acylated ghrelin in humans. J Nutr 136, 1533-1538

46. Serrano J, Casanova-Marti A, Depoortere I, et al. (2016) Subchronic treatment with grape-seed phenolics inhibits ghrelin production despite a short-term stimulation of ghrelin secretion produced by bitter-sensing flavanols. Mol Nutr Food Res 60, 2554-2564.
47. Serrano J, Casanova-Marti A, Gil-Cardoso K, et al. (2016) Acutely administered grape-seed proanthocyanidin extract acts as a satiating agent. Food Funct 7, 483-490.

48. Gruendel S, Garcia AL, Otto B, et al. (2007) Increased acylated plasma ghrelin, but improved lipid profiles 24-h after consumption of carob pulp preparation rich in dietary fibre and polyphenols. Br J Nutr 98, 1170-1177.

49. Gruendel S, Otto B, Garcia AL, et al. (2007) Carob pulp preparation rich in insoluble dietary fibre and polyphenols increases plasma glucose and serum insulin responses in combination with a glucose load in humans. Br J Nutr 98, 101-105.

50. Wanders AJ, van den Borne JJ, de Graaf C, et al. (2011) Effects of dietary fibre on subjective appetite, energy intake and body weight: a systematic review of randomized controlled trials. Obes Rev 12, 724-739.

51. Shin HS, Kindleysides S, Yip W, et al. (2015) Postprandial effects of a polyphenolic grape extract (PGE) supplement on appetite and food intake: a randomised dose-comparison trial. Nutr J 14, 96-104.

52. Yilmazer-Musa M, Griffith AM, Michels AJ, et al. (2012) Grape seed and tea extracts and catechin 3-gallates are potent inhibitors of alpha-amylase and alpha-glucosidase activity. J Agric Food Chem 60, 8924-8929.

53. Panickar KS (2013) Effects of dietary polyphenols on neuroregulatory factors and pathways that mediate food intake and energy regulation in obesity. Mol Nutr Food Res 57, 34-47.

54. Sies H, Stahl W \& Sevanian A (2005) Nutritional, dietary and postprandial oxidative stress. J Nutr 135, 969-972.

55. Cassidy A \& Minihane AM (2017) The role of metabolism (and the microbiome) in defining the clinical efficacy of dietary flavonoids. Am J Clin Nutr 105, 10-22.

56. Neveu V, Perez-Jimenez J, Vos F, et al. (2010) PhenolExplorer: an online comprehensive database on polyphenol contents in foods. Database 2010, bap024. 